\title{
Georg Wilhelm Steller und die moderne Flora der Kommandeur-Inseln
}

\author{
Gennady Firsov \& Olga Mochalova
}

\begin{abstract}
In a mission of the Russian Academy of Sciences, Georg Wilhelm Steller participated on the Second KamchatkaExpedition, led by Vitus Bering. Steller contributed outstanding findings to the fauna and flora of Russia. After being shipwrecked, he stayed for 9 months on the Bering Island and wrote a flora of that region, which continues to be important. This paper summarizes STELLER's life, the expedition and the flora of the Commander Islands.
\end{abstract}

\section{Zusammenfassung}

Der Deutsche Georg Wilhelm Steller nahm im Auftrag der russischen Akademie der Wissenschaften an der Zweiten Kamtschatka-Expedition unter Führung von Vitus BerING teil. Er lieferte hervorragende Erkenntnisse zur Flora und Fauna Russlands. Nach einem Schiffbruch erstellte er während eines 9-monatigen Aufenthaltes auf der Beringinsel (Kommandeur-Inseln) eine Flora dieses Gebietes, die noch heute von Bedeutung ist. STELlers Lebenslauf, die Expedition sowie die Flora der Kommandeur-Inseln werden vorgestellt.

\section{Georg Wilhelm Steller, bedeutender Naturforscher im 18. Jh.}

Der Deutsche Georg Wilhelm Steller (10. März 1709-12. November 1746) ist in die Wissenschaftsgeschichte Russlands als bedeutender Naturwissenschaftler und Forschungsreisender eingegangen. In Deutschland wird er jedoch eher weniger beachtet, weshalb in diesem Beitrag seine Lebensgeschichte und seine große Forschungsreise nach Kamtschatka und Alaska vorgestellt werden sollen. Die erste umfassende Biografie Stellers wurde im Jahr 1870 von Peter Pekarsky publiziert. Um die Wende vom 19. zum 20. Jh. ging der Norweger LeONHARD Steineger den Spuren Stellers nach. Er selbst begab sich nach Alaska, um ein möglicherweise doch noch überlebendes Exemplar der als ausgerottet geltenden STELLERschen Seekuh zu entdecken, was ihm jedoch nicht gelang. Er bezeichnete Steller als einen Pionier der naturwissenschaftlichen Erforschung Alaskas (STEINEGER 1936). Bücher und Manuskripte, die erst nach STELLERs Tod veröffentlicht wurden, erforschte und bearbeitete ausführlich auch D. I. Litvinov vor knapp 100 Jahren (1909).

Später beschäftigte sich V. I. LIPSKY mit STELLERS wissenschaftlichen Aktivitäten. Seine Ergebnisse wurden im Rahmen des 200-jährigen Bestehens des Botanischen Gartens in St. Petersburg zusammengetragen. Ein guter Eindruck von der Person G. W. Steller ist den in den letzten Jahren in Halle erschienenen Reisetagebüchern, Briefen und Dokumenten zu entneh- men. Leider ist jedoch keine Abbildung von STELLER überliefert.

\section{Von Deutschland nach St. Petersburg}

Georg Wilhelm Steller (eigentlich Stöller) wurde im fränkischen Windsheim als Sohn eines Kantors und Organisten geboren. Er wuchs in einfachen Verhältnissen auf, erhielt jedoch nach dem Besuch des Gymnasiums ein Stipendium, um in Wittenberg Theologie zu studieren. Als ihm aus finanziellen Gründen das Stipendium gestrichen wurde, nutzte er die Gelegenheit, das ihm ungeliebte Theologiestudium abzubrechen und sich der Medizin und den Naturwissenschaften zu widmen. Er studierte in Leipzig, Jena, Halle und Berlin. 1734 legte er sein Examen als Arzt ab. Für ihn gab es keine Aussicht auf eine wissenschaftliche Karriere in Preußen, weshalb er sich entschied, nach Russland auszuwandern, um dort sein Glück zu finden. Da er nur wenig Geld besaß, schloss er sich in Danzig als Wundarzt dem russischen Heer an und gelangte mit den Soldaten auf einem Lazarettschiff nach St. Petersburg. Er merkte dabei, dass sein Name für die Russen schlecht aussprechbar war, weshalb er sich in STELLER umbenannte.

Erzbischof Theophan Prokopovitch wurde in St. Petersburg auf ihn aufmerksam und gleichzeitig sein Gönner, da er persönlich sehr an Naturwissenschaften interessiert war. Er führte ihn in akademische Kreise ein. STELler knüpfte dabei u. a. Kontakte zu JoHAnn Amman, 


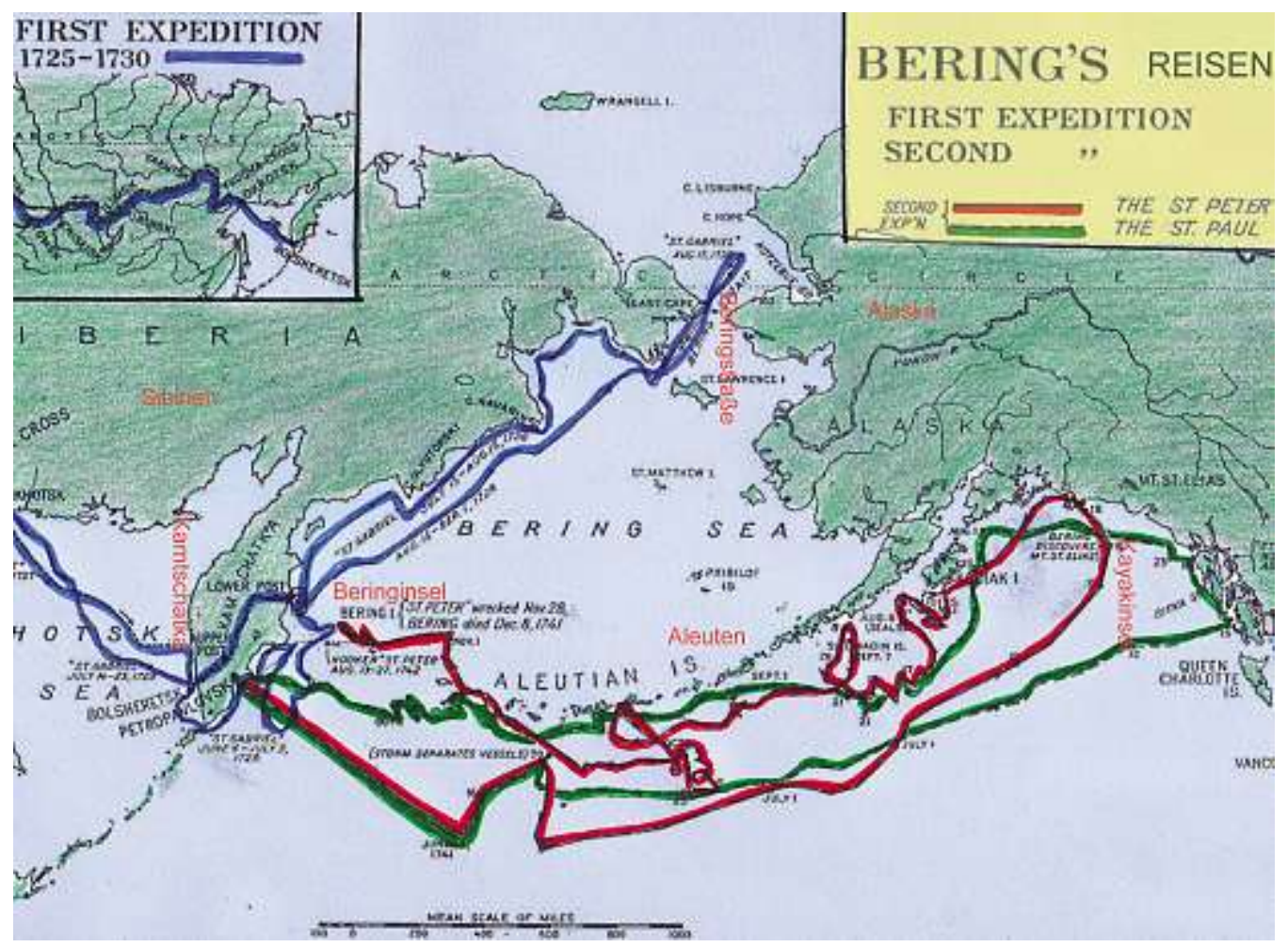

mit dem zusammen er intensiv botanisierte. Amman leitetet im Auftrag der Akademie der Wissenschaften den Botanischen Garten in St. Petersburg, in dem Pflanzen, die auf wissenschaftlichen Expeditionen gesammelt worden waren, kultiviert wurden. Die Hauptaufgabe des Botanischen Gartens und anderer privater Sammelgärten russischer Wissenschaftler bestand im 18. Jh. darin, so viele Wildpflanzen wie nur möglich zu kultivieren. Neue Arten wurden anhand der in den Gärten gehaltenen Pflanzen wissenschaftlich beschrieben. Auch der berühmte Professor und Sibirienforscher GMELIN publizierte viele solcher "Gar-

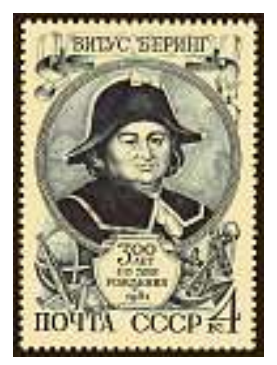
tenpflanzen-Beschreibungen " in seiner "Flora Sibirica“. Einige St. Petersburger Botaniker waren aber auch daran interessiert, auf Expeditionen gesammelte Pflanzen weiter zu verteilen und gaben Samen, Pflanzen sowie Her- barbelege an andere Sammlungen außerhalb der russischen Hauptstadt weiter.

Stellers Kontakt zu Amman und dem botanischen Garten ließ sicherlich verstärkt den Wunsch wachsen, sich selbst an Sammelreisen $\mathrm{zu}$ beteiligen. Das Interesse, an einer wissenschaftlichen Expedition teilzunehmen, wurde zudem durch Brigitta-Elena, Witwe von Daniel Messerschmidt, einem bedeutenden Forschungsreisenden des 18. Jh. (vgl. Firsov 1999), geweckt. STELLERs Wunsch ging in Erfüllung, denn im Jahr 1937 wurde er als Mitglied der Großen Nordischen Expedition (auch Zweite Kamtschatka-Expedition genannt), bestellt.

Abb. 1 (oben): Fahrtrouten der beiden von Bering geleiteten Kamtschatka-Expeditionen. Blaue Markierung: Erste Kamtschatka-Expedition. Rote Markierung: Route des Schiffes St. Peter, auf dem sich Steller und Bering aufgehalten haben. Grüne Markierung: Route des Schiffes St. PAUl.

Abb. 2 (unten): Russische Briefmarke mit der Darstellung von Vitus BERING. 


\section{Auf Expedition}

Außer ihm nahmen im Laufe der Jahre 800 weitere Personen an der von 1732-1743 dauernden Expedition teil, die von Vitus BerING organisiert wurde. Zur Vorbereitung waren STELLER die Tagebücher und Manuskripte des deutschen Sibirien-Forschers Messerschmidt sehr hilfreich. Kurz vor Antritt seiner Reise heiratete Steller Brigitta-Elena. Die Ehe hielt jedoch nur kurz: Als STELLER gerade erst auf dem Weg über Moskau nach Sibirien war, trennten sich beide wieder voneinander.

Im Herbst 1738 traf STELler in Tomsk ein, wo er sich mit den Wissenschaftlern MüLler und GMELIn traf. Nach Angaben seiner Begleiter war er ein fleißiger, zufriedener und genügsamer Mensch, der sich seine Mahlzeiten selber kochte und alle erdenklichen Entbehrungen auf sich nahm, wenn er dafür wissenschaftliche Feldforschungen betreiben konnte. Im folgenden Jahr schrieb er die „Flora Irkutensis“, in der 1152 Arten genannt wurden. GMELIN nahm später mehrfach auf sie in der „Flora Sibirica“ Bezug. Stellers Sammlung sibirischer Pflanzen wurde zudem für die Bearbeitung der „Flora Russica“ herangezogen. Am 21. September $1740 \mathrm{kam}$ STELler auf Kamtschatka an. Die Halbinsel erkundete er zusammen mit den Botanikern Krasheninnokov und Gmelin.

$\mathrm{Zu}$ Beginn des Jahres 1741 wurde er von dem in russischen Diensten stehenden Dänen VITUs BERING aufgefordert, statt eines zurückgetretenen Expeditionsarztes mit auf die Fahrt nach Amerika zu gehen. Bering war im Jahr 1728 bereits schon einmal von Kamtschatka aus auf eine Expedition aufgebrochen, auf der er die als Beringstraße bezeichnete Meerenge zwischen Sibirien und Alaska entdeckte. Er lieferte damit den Beweis, dass Amerika und Asien nicht miteinander verbunden sind. STELLER zögerte zunächst, willigte dann aber ein und wurde dadurch zum einzigen Botaniker seiner Zeit aus Russland, der auch Amerika besuchen und in Alaska

Abb. 3 (oben): Bunte Blumenwiese auf der Beringinsel.

Abb. 4 (unten): Maiglöckchen-Zweiblatt (Listera convallarioides), eine der seltenen Orchideen der Kommandeur-Inseln.
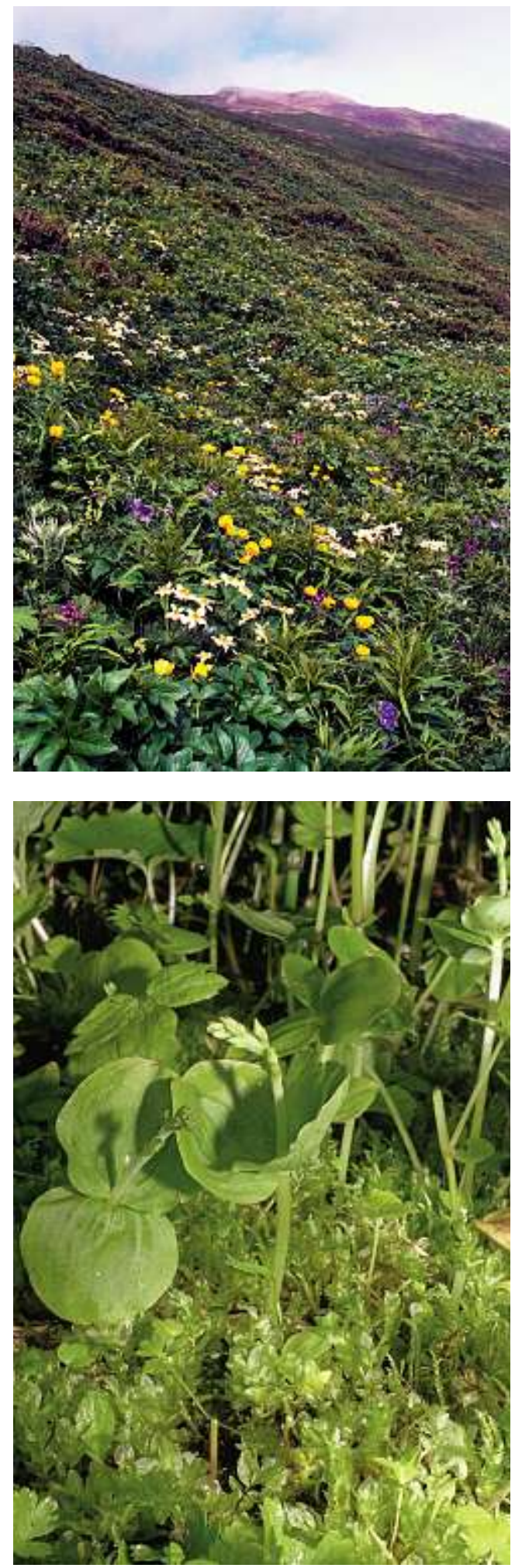

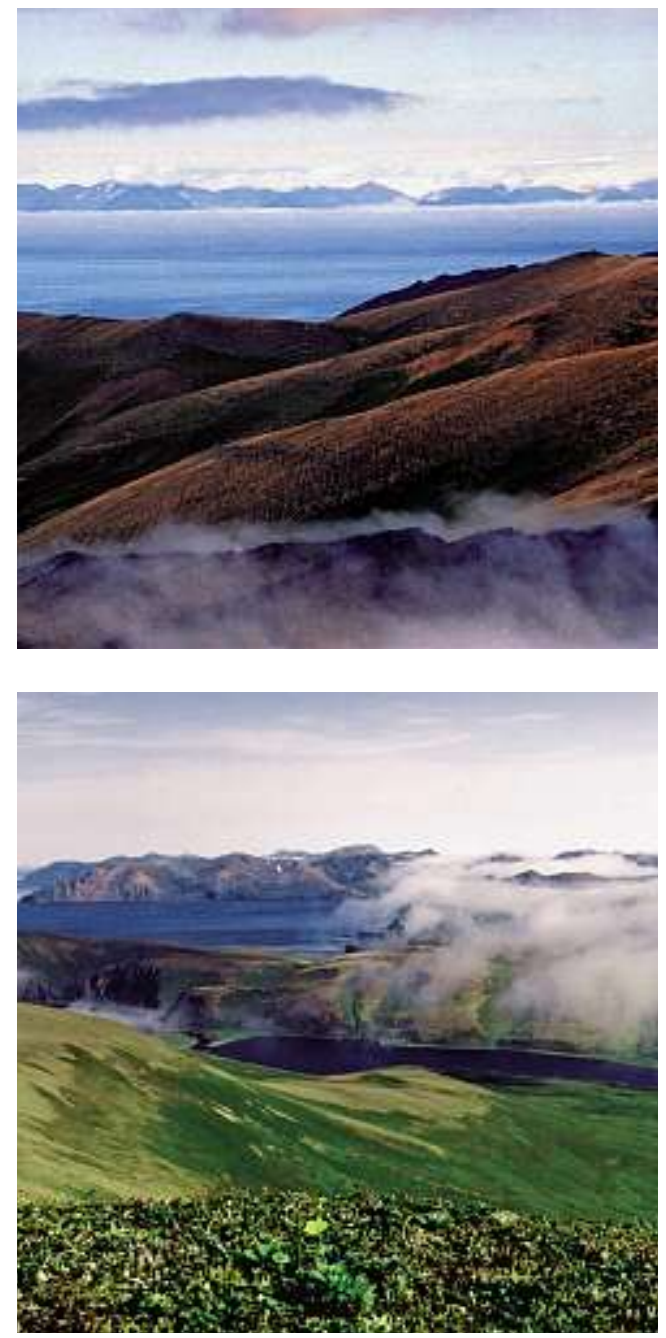

Pflanzen und Tiere sammeln würde. Zudem war er der erste Forschungsreisende, der den nördlichen Pazifik nördlich von Japan und Kalifornien studierte. Wichtige Resultate von StelLers Forschungsaufenthalten waren die Erkundung Nordwestamerikas, Nordsibiriens sowie der Kurilen.

Zur Expedition gehörten zwei Schiffe, das Gepäckschiff St. Peter, auf dem sich Bering und Steller aufhielten, sowie St. Paul. Nach technischen und navigatorischen Schwierigkeiten verloren die beiden Schiffe bald ihren Kontakt zueinander. Das Verhältnis zwischen Steller und Bering war leider nicht ungetrübt. Immer wieder kam es zu Auseinandersetzungen, weil STELLER kaum die Zeit dafür gegeben wurde, bei Landgängen die Gebiete genauer zu erkunden. Auf der neu entdeckten Kayak-Insel (früher St.-Elias-Insel), unweit der Westküste Alaskas mit dem Bering-Gletscher, hatte er beispielsweise nur zehn Stunden Zeit zum Botanisieren, weil BERING dort nur frisches Trinkwasser an Bord nehmen wollte. STELLER kritisierte, dass es doch keinen Sinn hätte, hier nur zu ankern, „um amerikanisches Wasser nach Russland zu bringen ". Trotzdem notierte STELLER dort in der kurzen Zeit über 150 Arten, darunter auch Lonicera caerulea, Cornus suecica, verschiedene Weidenarten sowie eine später als Rubus spectabilis beschriebene Brombeere. Diese wollte er als Kübelpflanze nach St. Petersburg schicken, um das attraktive Gewächs dort als Zierpflanze weiter zu vermehren. Der von STELLER verfasste "Catalogus Plantarum" ist die erste wissenschaftliche Abhandlung über ein Gebiet in Alaska. PaLlas veröffentlichte später (1781-1793) diese Manuskripte in den „Neuen Nordischen Beyträgen“. Neben den Pflanzen entdeckte StelLeR auch ein Lager mit Gebrauchsgegenständen und Schmuck der Alëuten-Eskimos. Er nahm einige Objekte im Tausch gegen eigene Gebrauchsgegenstände für seine ethnologische Sammlung mit.

\section{Gestrandet auf der Beringinsel}

Der weitere Verlauf der Expedition stand unter ungünstigen Zeichen: Skorbut, Stürme und Schiffbruch forderte das Leben vieler Mannschaftsmitglieder. Am 5. November 1741 strandete das Schiff St. Peter auf einer Insel, die später Beringinsel genannt und zur Inselgruppe der Kommandeur-Inseln gezählt wurde. Kurz darauf, im Dezember, starb Kommandeur VItus BERING auf „seiner" Insel an Unterkühlung. GeOrg STELLER ließ sich durch dieses tragische Unglück nicht von seinen Aktivitäten abhalten. Während des etwa 9-monatigen Zwangsaufenthaltes auf der Beringinsel organisierte er zusammen mit Leutnant WAXELL das Lagerleben. Das

Abb. 5 (oben): Morgenstimmung auf der Beringinsel.

Abb. 6 (unten): Auf den Kommandeur-Inseln ist es oft bewölkt, windig und regnerisch. 
Überleben war nur möglich, weil Robben, Otter und Seevögel leicht erlegt und als Nahrung verwendet werden konnten. Trotz der harten Rahmenbedingungen, u. a. auch aufgrund des schneereichen, nebeligen und stürmischen Wetters, untersuchte STELLER eine komplette Vegetationsperiode und notierte 218 Arten von Algen und Blütenpflanzen, darunter Primula cuneifolia, Rhododendron camtschaticum, Viola langsdorfii und Fritillaria camtschatcensis. 1906 wurde von B. Fedtschenko auf Grundlage des von STELLER verfassten „Catalogus Plantarum in Insula Beringii observatarum" die Flora der Kommandeur-Inseln veröffentlicht.

Neben den Pflanzen interessierte er sich sehr für die Tierwelt der Insel. Er beschrieb Seelöwen, diverse Vögel und die STELLERsche Seekuh (Hydrodamalis gigas $=$ Rhytina stelleri $=R h$. borealis), wodurch er zu weltweitem Ruhm kam. Denn bevor andere Wissenschaftler überhaupt die Gelegenheit hatten, diesen großen Meeressäuger zu sehen, war die Seekuh bereits 26 Jahre nach ihrer Entdeckung von Pelztierjägern ausgerottet worden. Nach der Rückkehr der Mannschaft nach Kamtschatka hatte sich nämlich die Kunde verbreitet, dass das Fleisch der Seekuh hervorragend schmecken würde, so dass sie gezielt für den Verzehr erlegt wurde. Ein weiteres Tier, das nach STELler benannt wurde, ist der Stellersche Seeadler (Haliaetus pelagicus), mit $2,5 \mathrm{~m}$ Flügelspannweite einer der größten Vögel Russlands. Auf der Beringinsel entstand auch seine viel beachtete Arbeit „De Bestiis Marinis“.

\section{Ende der Kamtschatka-Expedition und STELlERs Tod}

Nach der Errichtung eines neuen Schiffes aus den Resten der St. Peter konnte die stark geschrumpfte Mannschaft unter Führung von Leutnant WAXELL im August 1742 die Insel verlassen. Mit der Ankunft von WAXELL, SPANBERG

Abb. 7 (oben): Gelbschopf-Papageientaucher (Fratercula cirrhata), einer der besonders attraktiven Seevögel auf den Kommandeur-Inseln.

Abb. 8 (unten): Zahlreiche Robben und Seelöwen bevölkern die Küsten der Beringinsel.
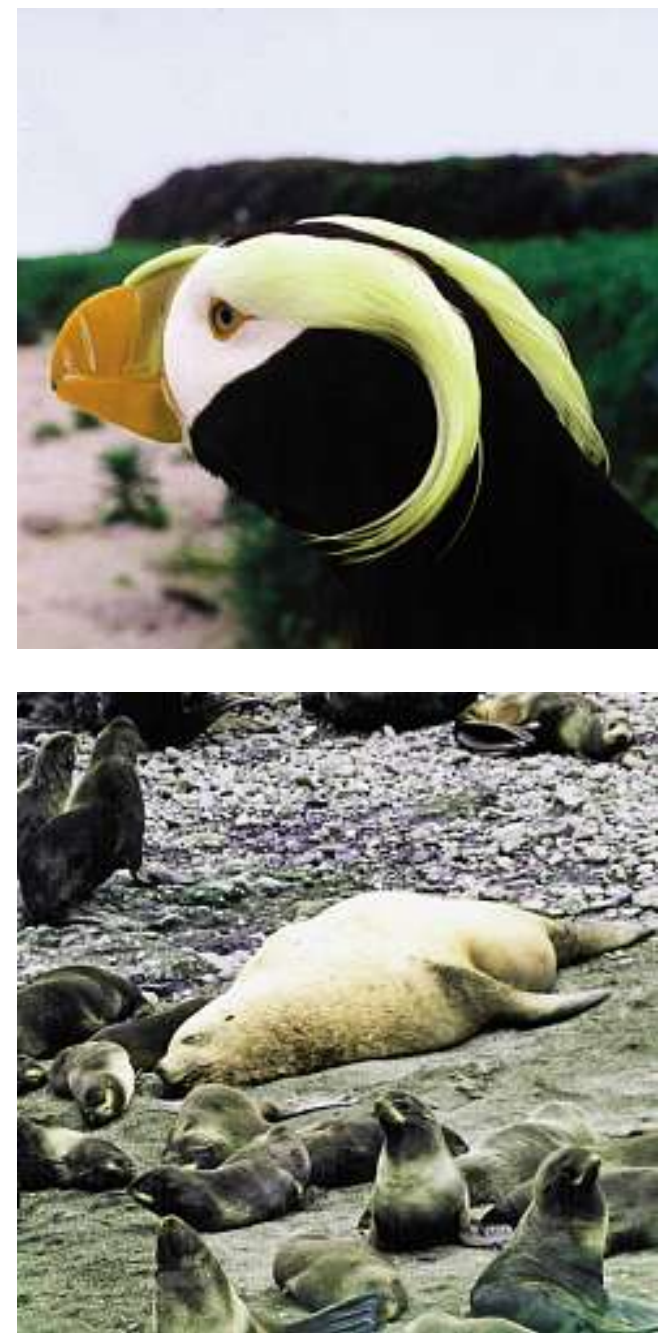

und Chirikov in St. Petersburg und der Nachricht von BERINGs Tod wurde die zweite Kamtschatka-Expediton offiziell für beendet erklärt.

STELLER wollte nicht sofort nach St. Petersburg zurückreisen und hielt sich deshalb anschließend noch zwei Jahre auf Kamtschatka und den Kurilen auf. Dort engagierte er sich u. a. auch für den Schutz der ortsansässigen Bevölkerung. Am 3. August 1744 wollte schließlich auch er, mit in 16 Kisten verpackten Sammlungen, in die Hauptstadt zurückkehren. Am 18. August 1746 schrieb er seinen letzten Brief an die Akademie der Wissenschaften, in dem er ankündigte, dass er im Frühling 1746 nicht in St. Petersburg eintreffen könnte, denn kurzfristig wurde 


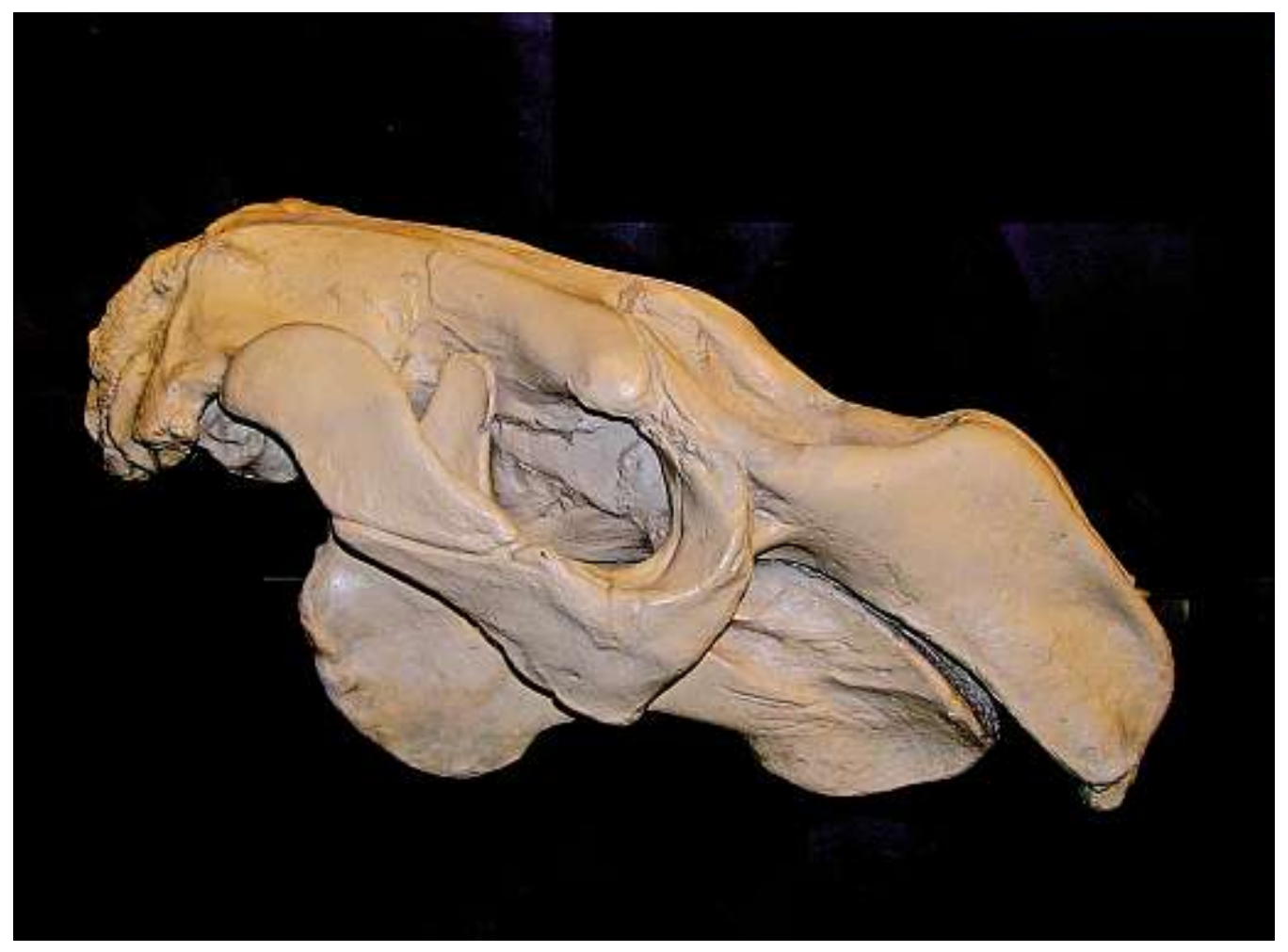

er in Solikamsk verhaftet. Ihm wurde vorgeworfen, die Bevölkerung in Ostsibirien aufgerufen zu haben, sich gegen die russische Herrschaft aufzulehnen und sogar Waffen verteilt zu haben. Er wurde jedoch wegen fehlender Beweise freigelassen, worauf hin er schnellstmöglich im sibirischen Winter aufbrach. Die Rückreise war sehr anstrengend, so dass er schwer erkrankte, eine Lungenentzündung bekam und starb. Erst über vier Monate nach seinem Tod erreichte der im August geschriebene Brief seine Adressaten in St. Petersburg.

\section{Interesse an Stellers Sammlungen}

Sowohl zu seinen Lebzeiten als auch später waren STELlers Sammlungen gefragt und stießen auch außerhalb von St. Petersburg auf großes Interesse. Etwa 80 seltene, von STELLER gesammelte Arten gelangten in Demidovs Garten in Solikamsk, in damaliger Zeit einer der reichsten Gärten Russlands. Und einige seiner Herbarbelege befinden sich noch heute im Herbarium von Peter Simon Pallas im British Museum in
London. Auch Carl v. Linné war sehr an STELLERs Sammlungen interessiert. Er erwartete in Schweden sehnlichst seine Rückkehr nach St. Petersburg und war neugierig auf die mitgebrachten Pflanzen.

Gregory Demodov, ein Schüler von Linné, kümmerte sich nach STELLERs Tod um einen Teil der Sammlung und die Manuskripte. Er schickte Duplikate von Pflanzenbelegen nach Schweden zu LinNÉ, um sie identifizieren bzw. neu beschreiben zu lassen. LinNÉs Schüler HaLENius veröffentlichte 1750 seine Dissertation „Plantae rariores Camtschatensis“, in der er 26 Arten aus Stellers, Krasheninnikovs und Gmelins Sammlungen beschrieb. 351 Arten dieser Kollektionen wurden auch in die „Species Plantarum“ aufgenommen. LiNNÉ würdigte GEORG STELler, in dem er eine Gattung aus der Familie der Thymelaeaceae Stellera nannte. Bereits kurz nach STELlers Tod publizierte S. P. KRA-

Abb. 9: Abguss des Schädels einer STELLERschen Seekuh. 
SHENINNIKOV (1750) ausführliche Beschreibungen der von LiNNÉ erstmals benannten Arten Thalictrum petaloideum (Heimat: Ferner Osten Russlands) und Alyssum hyperboreum (Heimat: Nördliches Nordamerika). Beide Arten waren aus von STEller gesammelten Samen im Botanischen Garten St. Petersburg herangezogen worden und geben Zeugnis von seiner großen Reise und beweisen, dass er tatsächlich, wenn auch nur kurz, in Amerika gewesen ist.

\section{STELlERs hervorragende Bearbeitung der Flora der Kommandeur-Inseln}

Die Untersuchungen STELLERs waren so gründlich und genau, dass sie noch in heutiger Zeit von Bedeutung sind. Die floristische Erkundung der Beringinsel wurde selbst nach 250 Jahren von keinem Botaniker in dieser Genauigkeit wiederholt. Einige Arten von Stellers Liste (z. B. Allocarya orientalis und Hypericum kamtschaticum) wurden erst am Ende des $20 \mathrm{Jh}$. auf der Insel wieder entdeckt.

\section{Aspekte der aktuellen Flora der Komman- deur-Inseln}

Die Kommandeur-Inseln sind die westliche Fortsetzung der Alëuten, von diesen allerdings durch einen sehr tiefen Meeresgraben klar abgetrennt. Beide Inselgruppen bilden die natürliche Abgrenzung zum Pazifik. Die Inseln sind der obere Teil eines riesigen vulkanischen Gebirgszuges, der sich über eine Länge von etwa $2000 \mathrm{~km}$ von Osten nach Westen erstreckt. Die KommandeurInseln liegen etwa $175 \mathrm{~km}$ östlich von Kamtschatka. Die beiden größten Inseln des Archipels sind die Beringinsel (1667 km² Fläche) und

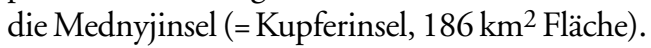
Erstere ist etwa $90 \mathrm{~km}$ lang und durchschnittlich $18 \mathrm{~km}$ breit, die Mednyjinsel hat eine Ausdehnung von etwa 53 x $5 \mathrm{~km}$. Die Landschaft auf diesen Inseln ist sehr abwechslungsreich mit hohen Gipfeln, Hügeln, Plateaus, Schluchten, Tälern, Flüssen und Bächen. Zudem weisen die Inseln eine große Artenvielfalt auf, weshalb sie unter Schutz gestellt wurden. Beeindruckend ist

Abb. 10: Natürliches Felstor auf der Beringinsel.

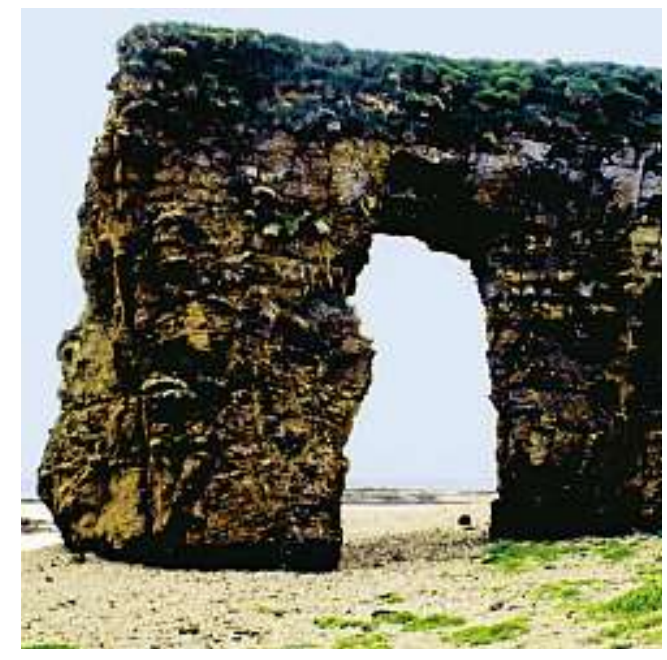

die Vielzahl der dort heimischen Meerestiere. So bieten die Inseln beispielsweise Brutplätze für über 600000 Seevögel.

Die Kommandeur-Inseln sind von pflanzengeografischer Bedeutung, weil sie eine wichtige Schnittstelle zwischen der asiatischen und amerikanischen Flora darstellen. Insgesamt hat die 432 Arten und Unterarten aus 200 Gattungen umfassende Flora starke Ähnlichkeit mit der Pflanzenwelt auf Kamtschatka. Mehrere Dutzend Arten haben auf den Kommandeur-Inseln ihre westliche Verbreitungsgrenze erreicht, andere dagegen kommen sonst nur noch weiter im Westen vor.

Wälder sowie größere Strauchgürtel fehlen auf den Kommandeur-Inseln aufgrund der häufigen Stürme, der relativ niedrigen Sommertemperaturen sowie des meist wolkenverhangenen Himmels und der dadurch reduzierten Strahlungsintensität.

Nur an manchen Stellen trifft man auf kleine, höchstens $2 \mathrm{~m}$ hohe Gehölze wie Salix alaxensis, Salix lanata oder Sorbus sambucifolia. In der Tundravegetation der Inseln gedeihen viele Stauden und Zwergsträucher, darunter zahlreiche Ericaceen wie Rhododendron aureum, Rh. camtschaticum, Arctous (Arctostaphylos) alpina, Cassiope lycopodioides, Vaccinium uliginosum und Bryanthus gmelinii. Auf den KommandeurInseln kommen 16 verschiedene Farnarten vor (z. B. Asplenium viride, Botrychium robstum etc.). Von den 14 Orchideenarten des Archipels ste- 


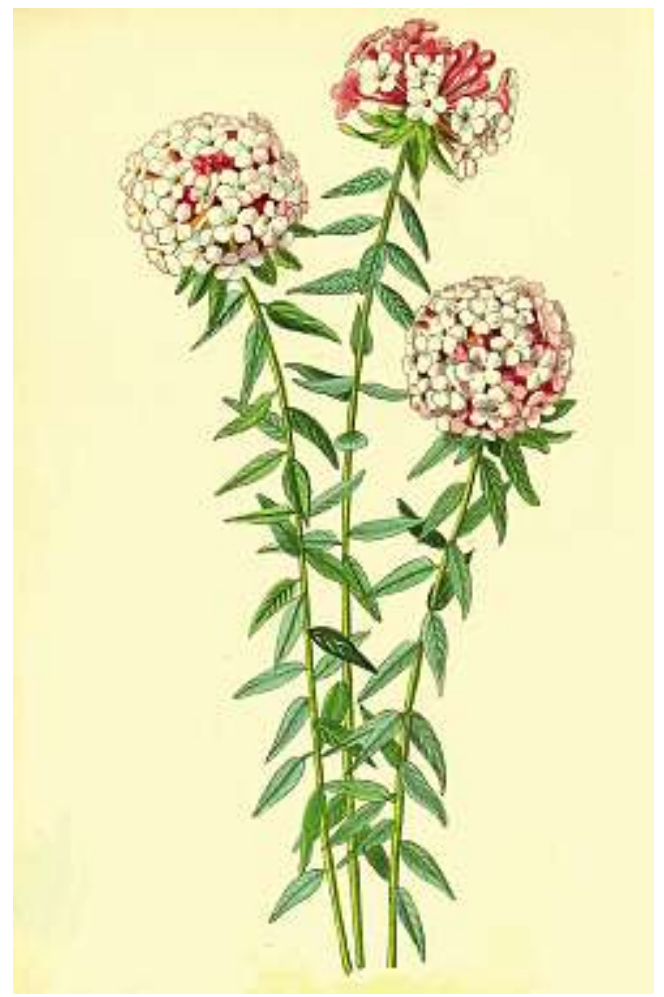

hen viele auf der Roten Liste. Ausgesprochen dekorative und gartenwürdige Arten sind Fritillaria camtschatcensis mit seinen schwarz-purpurnen Blüten und Streptopus amplexicaulis (Asparagaceae), dessen Beeren im Herbst rot leuchten. Weitere auffällige Arten sind Campanula lasiocarpa und Delphinium brachycentrum. Die Früchte von Lonicera caerula sind ungiftig, schmecken nicht bitter und werden von den Heimischen gegessen.

In der Kamtschatka-Region gibt es leider keine botanischen Gärten, um die hier vorkommenden attraktiven Arten auf ihre Kulturbedingungen hin zu überprüfen, wie es bereits STELLER beabsichtigte. Viel und langwierige Arbeit muss noch von Botanikern und Gärtnern geleistet werden, um Pflanzen der Kommandeur-Inseln in die Kultur einzuführen. Es wäre also wünschenswert, wenn die vor über 250 Jahren zuerst von STELler untersuchte Flora der KommandeurInseln noch gründlicher bearbeitet würde und somit die Kenntnisse über sie aktualisiert werden könnten. Es sollte zudem nachgeholt werden, diejenigen Pflanzen aus Stellers Liste zu sammeln, die bisher in keiner Sammlung oder Garten vorhanden sind.

STELLER, seine Reise sowie seine hervorragenden Untersuchungen zur Flora Russlands und Alaskas sollten auf keinen Fall in Vergessenheit geraten. Immerhin ist die Kommandeur-Bucht auf der Beringinsel, von dem aus das wiederhergestellte Schiff St. Peter zusammen mit Steller von Alaska zurück nach Russland aufbrach, eine touristische Attraktion, die von nahezu allen Kreuzfahrtschiffen in dieser Region angelaufen wird.

\section{Literatur}

Fedtschenko, B. F. 1906: Flore des îles du Commandeur. - Krakau.

Firsov, G. A. 1999: Daniel Gottlieb Messerschmidt - Forschungsreisender und Pflanzensammler in Russland. - Palmengarten 63: 62-64.

Gmelin, J. G. 1747-1769: Flora sibirica sive historia plantarum sibiricae. Vol. 1-4 - Petropoli.

Hintzsche, W., Nickol, T. \& Novochatko, O. (Hrsg.) 2000: Georg Wilhelm Steller - Briefe und Dokumente. Band 1. 1740. - Halle.

Hintzsche, W. 2000: Georg Wilhelm Steller, Stepan Kraseninnikov, Johann Eberhard Fischer. - Reisetagebücher. Bd. 2. 1735-1743. Unter Mitarbeit von Nickol, T., Novochatko, O. \& Schulze, D. - Halle.

Hintzsche, W. 2000: Georg Wilhelm Steller. Briefe und Dokumente. Bd. 3. 1739. Unter Mitarbeit von Nickol, T., Novochatko, O. \& Schulze, D. - Halle. Pallas, P. S. 1784-1788: Flora rossica seu stirpium imperii rossici per Europam et Asiam indigenarum descriptiones et icones. T. 1, Pars 2. - Petropoli. Pallas, P. S. 1781-1793: Neue nordische Beyträge zur physikalischen und geographischen Erd- und Völkerbeschreibung. Naturgeschichte und Ökonomie. Bd. 2, 5, 6. - St. Petersburg, Leipzig.

Steineger, L. 1936: Georg Wilhelm Steller, the pioneer of Alaskan natural history. - Cambridge, Massachusetts.

\section{Internetseiten:}

http://www.vulkaner.no/t/kamchat/steller.html (abgerufen am 10.2. 2006) http://de.wikipedia.org/wiki/Georg_Wilhelm_Steller (abgerufen am 10.2.2006)

Abb. 11: Stellera chamaejasme, Darstellung aus Curtis' Botanical Magazine, Nr. 9028. 